\title{
Disambiguation in conversation: the case of disambiguating parentheticals
}

\section{Stefano Predelli ${ }^{1}$}

Received: 18 February 2021 / Accepted: 27 August 2021 / Published online: 12 September 2021

(c) The Author(s) 2021

\begin{abstract}
This essay presents an analysis of the conversational role of disambiguation, with special attention to disambiguating parentheticals such as 'bats, the furry animals, are not easy to find'. The essay proposes an enriched representation of conversational states as pairs of an interpretation function and standard common belief, it represents disambiguations within the ensuing framework, and, on the basis of these conceptual tools, it proposes a systematic picture of the updates achieved by disambiguating parentheticas.
\end{abstract}

Keyword Conversation · Update · Disambiguation

In what follows, I propose a formal framework for the conversational role of disambiguation, with a particular focus, in the second half of this essay, on the case of what I call disambiguating parentheticals, as in:

(1) bats, the furry animals, are not easy to find

(2) I bought a bat (a pipistrelle).

Section one sketches a simple framework for the conversational updates engendered by (assertoric, unchallenged, etc.) utterances in an unambiguous language. Section two adapts this framework to the case of (lexically) ambiguous languages, and it introduces disambiguations as elements of conversational states. Section three presents the main concepts in my analysis of the conversational role of disambiguation: the ideas of utterance-disambiguation and of updated disambiguation. After a few informal hints on supplements in section four, section five applies my apparatus to the case of disambiguating parentheticals such as (1) and (2).

A small caveat about my scenarios is in order before I begin. As evidenced by (1) and (2), I take the noun 'bat' as the protagonist in my colloquial examples. In

Stefano Predelli

Stefano.predelli@nottingham.ac.uk

1 Department of Philosophy, University of Nottingham, Nottingham NG72RD, UK 
order to simplify my informal commentaries, I focus on the contrast between its two most common interpretations, namely as a noun for certain wooden implements, or as a noun designating the members of the order Cheiroptera. For brevity's sake, I also occasionally gloss these readings simply in terms of, respectively, 'stick' and 'pipistrelle', setting aside the fact that, in proper zoological jargon, 'pipistrelle' is used to designate a subspecies of Cheiroptera.

\section{Conversing in an unambiguous language}

When you speak or write, I may occasionally focus my attention on the sounds or scribbles you produce-if, for instance, I am absorbed in your tone of voice or in your calligraphic skills. When we are engaged in a conversation, however, our interest must be wider: those sounds or scribbles are tokened so as to exemplify fully-fledged expressions and, as a result, to convey a meaning.

According to a standard format, an expression may be represented (at least for some purposes) as a triple consisting of a phonological form (here, for convenience's sake, a string of letters), a syntactic category, and a meaning. As in, say: $d o g=<$ 'dog', noun, being a dog $>$, where, for simplicity's sake, a noun's meaning is presented directly as a property. ${ }^{1}$ A language may then be identified (at least for some purposes) with a collection of the expressions in its lexicon, together with the phonological, syntactic, and semantic regularities for the formation of complex affairs. Often leaving these latter elements as implicit, I represent a language $L$ simply as a class of lexical expressions, as in, say, $\operatorname{dog} \in \operatorname{Eng}(\text { lish })^{2}$

According to a common way of speaking, some words are ambiguous, in the sense that 'they have more than one meaning'. Of course, if words are regimented as expressions in the aforementioned sense, there can be no ambiguous words: trivially, for all forms $s$, categories $k_{1}, k_{2}$, and meanings $m_{1}, m_{2}$, if $m_{1} \neq m_{2}$ then $<s, k_{1}, m_{1}>\neq<$ $s, k_{2}, m_{2}>$. It will thus do to speak of ambiguous forms instead, as in the idea that the three-letter form 'bat' may serve as the form of two different English expressions:

(a) bat $_{1}$ : < 'bat', noun, being a pipistrelle >

$$
\text { bat }_{2}:<\text { 'bat', noun, being a stick > . }
$$

Officially, then, a lexical form $s$ is ambiguous in $L$ iff $<s, k_{1}, m_{1}>\in L$ and $<s, k_{2}$, $m_{2}>\in L$, where $k_{1} \neq k_{2}$ or $m_{1} \neq m_{2} .^{3}$

Let a lexical form $s$ be admissible in $L$ iff $\exists k, m:\langle s, k, m\rangle \in L$. Then, $L$ is an unambiguous language iff no $s$ admissible in $L$ is ambiguous. So, an unambiguous language $L$ determines an interpretation function $\lambda_{L}$ from (lexical) forms to meanings:

\footnotetext{
1 See for instance (Potts, 2007) and (Kracht, 2007). More appropriately, meanings may be represented by more fine-grained affairs, such as, say, Kaplan-style characters, namely functions which (given a context) yield a content. The complications related to context-sensitive expressions and, more generally, to the proper format for the representation of meaning are nevertheless of no immediate relevance for what follows.

2 My allusion to English as the language of choice facilitates my presentation, but it may safely be substituted with alternative locutions pertaining to varieties of English, to dialects, or to idiolects.

3 Note that, for simplicity's sake, my focus in what follows is restricted to ambiguous expressions of the same category, as in the two senses of 'bat', rather than in the uses of, say, 'well' as a noun or an adverb.
} 
$\lambda_{L}=\{\langle s, m\rangle: \exists k:\langle s, k, m\rangle \in L\} .{ }^{4}$ For instance, if 'dog' is a form in an unambiguous fragment of English, then that fragment's interpretation function uniquely associates that form with a particular meaning, such as the property of being a dog.

Unambiguous languages allow for a simple representation of the conversational effects of an utterance. As before, take on board the language's phonological, syntactic and semantic regularities for the formation of complex expressions, and leave issues of syntactic ambiguity aside. Then, a token of a sentential form $S$ containing exactly the lexical items $s_{1} \ldots s_{n}$ is associated by $\lambda$ (subscripts omitted for legibility's sake) with the unique proposition $\lambda(S)=\mu\left(\lambda\left(s_{1}\right) \ldots \lambda\left(s_{n}\right)\right)$, where $\mu$ is the semantic effect of $S$ 's structure. For instance, the presumably unambiguous English sentential form ' $a$ dog is barking' is mapped with the proposition that a dog is barking. Consequently, as I am about to explain, your awareness of that token and your linguistic competence suffice for recovering the the proposition that a dog is barking-for instance, in cases of assertoric scenarios, as a proposed enrichment of common belief. ${ }^{5}$

Since utterances are manifest events (in the sense of Stalnaker, 2014), the assumption of shared competence with an unambiguous $L$ suffices to put the proposition expressed by the uttered sentential form 'on the conversational record', roughly in the sense that, if the utterance is accepted, that proposition becomes part of common belief.$^{6}$ I sharpen this idea with the help of some symbolism. Let a conversational state $\sigma$ for an unambiguous language $L$ be a pair consisting of an interpretation function $\lambda_{\sigma}\left(=\lambda_{L}\right)$ and a set of propositions $\beta_{\sigma}$, where $\lambda_{\sigma}$ reflects the conversants' shared linguistic competence with $L$, or, at least, with the aspects of it that matter at that stage, and $\beta_{\sigma}$ is the set of common beliefs in $\sigma$ (subscripts in ' $\lambda_{\sigma}$ ' and ' $\beta_{\sigma}$ ' hereinafter omitted when irrelevant or obvious). As long as an utterance $u_{S}$ of $S$ is publicly available to all conversants, and as long as it is common belief among them that $\lambda$ is the appropriate interpretation function at that stage, what is thereby also publicly available is the proposition $\lambda(S)$, that is, the proposition expressed by $S$ according to $\lambda$. Then, the effect of an utterance $u_{S}$ on a state $\sigma=\langle\lambda, \beta\rangle$ is the enrichment of $\beta$ with that proposition:

(b) $\sigma\left[u_{S}\right]=<\lambda, \beta \cap \lambda(S)>.^{7}$

\footnotetext{
${ }^{4}$ I employ $f$ as designating both the graph of a function $f$, that is, a set of pairs $\langle x, y\rangle$, and the function itself, as in $f(x)=y$.

5 The background is provided by the classic Stalnaker-inspired framework for conversational updates, see for instance (Stalnaker, 1999) and the considerable ensuing literature; see Farkas and Bruce (2009) for the idea of a proposed update. Here as before, I restrict my attention to simple assertoric, literal utterances of indicative sentences, leaving aside the tangential and independent enrichments required for the treatment of different types of sentences and/or speech-acts.

${ }^{6}$ Stalnaker defines a manifest event as 'something that happens in the environment of the relevant parties that is obviously evident to all. ... In such a case, it immediately becomes common knowledge that the event has happened' (Stalnaker, 2014: p. 47). See also the discussion of copresence in Clark and Marshall (1981) and the analysis of its relationships with other accounts of mutual belief in Barwise (1988).

7 As usual, with the obvious qualification omitted, as in the implicit restriction to asserted indicative sentences that are subsequently accepted by the conversants. Also: in my informal presentation, I occasionally speak of the 'addition' of a proposition to common belief, and of as 'growing' accordingly. Of course, in the formal representation of propositions as sets of possible worlds, it 'shrinks' as a result of non-trivial intersections.
} 
For instance, the effect of your utterance of the English sentence 'a dog is barking' is that of intersecting our antecedent common beliefs with $\lambda_{E n g}$ ('a dog is barking'), namely with the proposition that a dog is barking.

\section{Updates with an ambiguous language: preliminaries}

Ambiguous languages, that is, languages with ambiguous admissible forms, complicate this picture. For instance, in the absence of further information, the manifest act of tokening the English form 'bat' does not make manifest a unique expression, and hence does not suffice for any contribution to propositional update. And so, as we commonly say, it makes no sense to ask for an out-of-the -blue interpretation of 'bat': what are proper objects of interpretations are either 'bat ${ }_{1}$ ' or 'bat 2 ', the expressions in (a). In the jargon from the previous paragraph, when $L$ is an ambiguous language, $\lambda$ is a relation, but it is not a function. A form admissible in an ambiguous language may then be associated with a particular meaning only on the basis of something over and above $\lambda$, namely on the basis of what we commonly call a disambiguation.

I represent a disambiguation of lexical forms as a 'pruning' of $\lambda$, in the following sense. Given an ambiguous lexical form such that $\left.\left.\left\{<s, m_{1}\right\rangle \ldots<s, m_{n}\right\rangle\right\} \subseteq \lambda$ (with $\left.m_{1} \neq \ldots \neq m_{n}, n>1\right)$, a lexical disambiguation $\delta_{s, i}$ of $s$ is such that.

(c) $\delta_{s, i}(\lambda)=\lambda-\left\{<s, m_{j}>\in \lambda: j \neq i\right\}$.

For instance, given the representation in (a), the disambiguation $\delta_{b a t, l}$ of the English ambiguous form 'bat' achieves the effect of obliterating the mapping of 'bat' with the property of being a stick: $\delta_{\text {bat, } 1}\left(\lambda_{E n g}\right)=\left\{<\right.$ 'bat', $m_{1}=$ pipistrelle $>,<$ 'bat', $m_{2}$ $=$ stick $>\}-\left\{<\right.$ 'bat', $\left.m_{2}>\right\}$. As a result, $\delta_{b a t, 1}\left(\lambda_{E n g}\right)$ uniquely associates 'bat' with $m_{1}$, so that all tokens of that form end up designating $\delta_{b a t, 1}\left(\lambda_{E n g}\right)$ ( 'bat'), namely the property of being a pipistrelle.

Taking on board the compositional regularities for the formation, pronunciation, and interpretation of complex expressions (and leaving aside issues of structural ambiguity), disambiguations allow for an extension of (b) to ambiguous languages. I present the general traits of this extension in the remainder of this section, before I proceed to some amendments in section three.

Given a language $L$ containing the ambiguous lexical forms $s_{1} \ldots s_{n}$, a disambiguation $\delta_{L}$ of $L$ is a set of lexical disambiguations $\left\{\delta_{i} \ldots \delta_{j}\right\}, 1 \leq i, j \leq n$ and $i \neq j$ (I avoid double subscripts and write ' $i$ ' for ' $s_{i}$ ' in ' $\delta_{I}$ '; I also omit the second subscript in ' $\delta_{s, m}$ ' for legibility's sake). A full disambiguation of $L$ is a disambiguation $\left\{\delta_{i}: 1 \leq i\right.$ $\leq n$ \}, that is, colloquially, a set of all the disambiguation needed for dealing with the language's ambiguous forms. Let a disambiguation $\delta$ of $L$ be adequate for a sentential form $S$ of $L$ containing occurrences of the ambiguous forms $s_{i} \ldots s_{j}$ iff $\left\{\delta_{i} \ldots \delta_{j}\right\} \subseteq$ $\delta$; and let a disambiguation $\delta$ adequate for $S$ be such that $\delta(\lambda)(S)=\mu\left(\delta_{i}(\lambda)\left(s_{i}\right), \ldots\right.$ $\delta_{j}(\lambda)\left(s_{j}\right)$ ), where, as before, $\mu$ is intended to reflect the compositional effects of $S$ 's (non-ambiguous) structure. So, if $\delta$ is an adequate disambiguation for a sentential form $S$, the function $\delta(\lambda)$ maps $S$ to a unique proposition; and if $\delta$ is a full disambiguation for $L, \delta(\lambda)$ is a function from all sentential forms in $L$ to propositions. 
Enrich the representation of a conversational state for an ambiguous language $L$ as in $\sigma=\langle\langle\lambda, \delta\rangle, \beta\rangle$, with $\lambda$ and $\beta$ as before, and $\delta$ a disambiguation for $L$. Then, the effects of an utterance $u_{S}$ of $S$ may preliminarily be recorded as follows. If $\delta$ is adequate for $S$,

(d) $\sigma\left[u_{S}\right]=<<\lambda, \delta>, \beta \cap \delta(\lambda)(S)>$.

For instance, take a disambiguation $\delta$ of 'bat' and 'bank', such that $\delta_{b a t}$ and $\delta_{\text {bank }}(\epsilon$ $\delta$ ) select respectively the zoological and monetary senses of those forms. Then, as the result of an utterance of the lexically ambiguous English sentential form 'a bat went to the bank', the antecedent common belief $\beta$ is enriched as in $\beta \cap$ that a pipistrelle went to a financial establishment, that is, it is enriched with the proposition expressed by that form on the basis of the disambiguation in question.

What if $\delta_{\sigma}$ is not adequate for $S$ ? In practice, communicative exchanges may well be rather tolerant in this respect: if only 'bat' is disambiguated, an utterance of 'a bat went to a bank' may perhaps suffice to intersect $\beta$ with, say, the proposition that a pipistrelle went somewhere. These independently interesting subtleties are nevertheless of no immediate significance for what is about to follow. I thus adopt without further ado the (independently harsh) simplification that conversational ambiguity fails to engender any propositional update whatsoever. ${ }^{8}$ That is, for my purposes (and only for those) it will do to assume that, if $\delta_{\sigma}$ is not adequate for $S$,

(e) $\sigma\left[u_{s}\right]=\sigma$.

Colloquially, all of the above reflects a certain initially intuitive picture of smooth communication (perhaps modulo the stipulation in (e)). Informally, what is required to communicate with an ambiguous language such as English is that (i) we share the linguistic competence required from English speakers, (ii) we have common access to appropriate disambiguating clues, and (iii) we share a set of common beliefs. Deciding what, in practice, establishes the disambiguation appropriate at a particular conversational stage, that is, determining what sort of clues matter for (ii), is, of course, a notoriously difficult issue. For my purposes here, much that is of relevance for this independently important question may safely be left to empirical investigations pertaining to the roles of the conversants' mental states, of the topic of conversation, of a variety of environmental factors, and more besides. For pedagogical perspicuity, and also in order to indirectly highlight my relative nonchalance on this issue, in what follows I occasionally settle for a simple-minded locational metaphor: when we converse at the zoo, so I write, 'bat' generally takes on the meaning of 'pipistrelle', whereas, if we are chatting at Yankee Stadium, it becomes associated with the meaning of 'stick'.

And yet, certain other aspects related to (ii) do matter for my aims, since these are features of a conversational state's disambiguation that bear interesting relations to that state's other components, and in particular with common belief. And so, they are aspects that matter from my viewpoint, namely for the analysis of the conversational role of disambiguations and, eventually, for the application of my framework to the case of disambiguating parentheticals. I proceed to their study in the next section.

\footnotetext{
8 In other words, for simplicity's sake, I focus on cases of full disambiguation and I dismiss cases of partial disambiguation (as when 'a bat went to the bank' is interpreted with respect to a disambiguation of 'bat', without an accompanying disambiguation of 'bank').
} 


\section{Disambiguations and updates}

In section two I flanked my presentation of (d) and my informal gloss with a repeated caveat: this is a preliminary depiction of the update engendered by an utterance in an ambiguous language. One point is worthy of note: (d), and for that matter (b), take a conservative approach towards a conversational state's first element, namely $\lambda$ in the case of (b) and $\langle\lambda, \delta\rangle$ in the case of (d). These approaches are conservative in the sense that, according to (b) and (d), the interesting effect of an update is solely that of intersecting $\beta$ with an appropriate proposition: the antecedent state $\sigma$ and the resulting state $\sigma\left[u_{S}\right]$ have the first element in common. For the purpose of what follows, I grant (merely for simplicity's sake) that this much is appropriate when it comes to $\lambda$, in the sense that we enter a conversation with a common assumption of linguistic competence, which we keep unchanged as we proceed. ${ }^{9}$

But the case with $\delta$ is of greater interest. Section two wrapped things up with a deliberately casual attitude towards typical sources of disambiguation. There, I simply gestured towards certain plausible candidates, and I eventually settled on a simple-minded metaphor: other things being equal, an utterance of 'bat' at the zoo is interpreted according to the zoological sense of that term, and it leaves the stick-related meaning aside. And yet, of course, even conversations taking place at the zoo may occasionally digress on baseball, and, on those occasions, 'bat' may end up taking on a different meaning than that related to pipistrelles. Once again, many of the reasons why this may be the case can safely be left aside for my purposes. But others are of significance as an informal background for the central ideas in this section: the notion of an utterance-disambiguation, and the related revision of the conservative stance summarized in the foregoing paragraph, namely the notion of updated disambiguation.

Suppose that, out of the blue and with no particular environmental clues in place, I say.

(3) bats locate obstacles by echolocation,

and that you correctly hypothesize that what I proposed was a proposition about pipistrelles. In abstract, I suppose, your hypotheses are defeasible. In principle, that is, I could perhaps have used that sentential form in order to express the absurd belief that wooden sticks employ certain methods for the location of objects, and I could have done so without thereby relinquishing my status of competent English speaker. Yet, as I am about to explain, there are reasons why this option would have been conversationally less than optimal.

The reasons in question depend on $\beta$, our antecedent stock of common beliefs. In general,

communication, whether linguistic or not, normally takes place against a background of beliefs or assumptions which are shared by the speaker and his

\footnotetext{
9 Incidentally, my discussion also often implicitly takes on board the unrealistic assumption that our competence is complete, in the sense that the domain of is the class of all admissible forms (as in the pretence that we enter a conversation under the assumption that any English sentence will fall under our common mastery of that language). Rectifying these unrealistic assumptions is an independently interesting task, but it may safely be left aside here.
} 
audience, and which are recognized by them to be so shared. ...The more common ground we can take for granted, the more efficient our communication will be. (Stalnaker, 1999: p. 48)

In a standard scenario for (3), then, communication efficiently proceeds on the basis of common beliefs such as the obvious facts that sticks are inanimate, that what locates obstacles is generally sentient, that pipistrelles are animals, and other affairs of that sort. Accordingly, the interpretation of (3) naturally adjusts in conformity with the immediate entailments of these beliefs: the speaker would have blatantly contradicted $\beta$ unless she intended to speak of pipistrelles, that is, unless she intended 'bat' to be disambiguated accordingly. In a nutshell, and in plain English: we generally disambiguate not only by taking into consideration the topic of conversation or the state of our surrounding environment, but also by appealing to common sense. That is, colloquially, we appeal, among other things, to certain aspects of the utterance in question - the facts that, say, the disambiguation of its form along baseball-related lines yields a preposterous result, and that its zoological disambiguation would nicely complement our antecedent understanding.

In what follows, I begin to sharpen these ideas by means of the idea of utterancedisambiguation. Let an adequate disambiguation $\delta$ of a sentential form $S$ containing exactly the ambiguous lexical form $s_{1} \ldots s_{n}$ be minimal iff $\delta=\left\{\delta_{1} \ldots \delta_{n}\right\}$. The utterancedisambiguation $v^{S, \sigma}$ engendered by an utterance of $S$ with respect to a state $\sigma=<$ $<\lambda, \delta>, \beta>$ is a minimal disambiguation such that.

(f) if for all disambiguations $\delta$ other than $\delta^{*}, \delta(\lambda)(S) \cap \beta=\emptyset$, then $v^{S, \sigma}=\delta^{*}$; otherwise $v^{S, \sigma}=\emptyset$.

So, an utterance-disambiguation engendered by $S$ is the unique disambiguation $\delta$, if any exists, such that disambiguating $S$ with $\delta$ is compatible with common belief. For instance, the utterance-disambiguation $v^{(3)}$ achieved by (3) is the unique disambiguation consistent with the aforementioned common-sense beliefs about bats, sentient beings, and sticks. Since the disambiguation that interprets 'bat' as designating sticks yields an absurd result, $v^{(3)}$ disambiguates that form in the zoological sense. ${ }^{10}$

Let $\sigma$ be a state $\langle\langle\lambda, \delta\rangle, \beta\rangle$ such that $\delta=\left\{\delta_{m} \ldots \delta_{n}\right\}$, let $S$ contain exactly the ambiguous lexical forms $s_{i} \ldots s_{j}$, and let $A=\{m \ldots n\} \cap\{i \ldots j\}$. Define the updated disambiguation $\psi^{S, \sigma}$ achieved on $\sigma$ by an utterance of $S$ as in.

(g) $\psi \stackrel{S, \sigma}{=}\left(\delta-\left\{\delta_{n}: n \in A\right\}\right) \cup v^{S, \sigma}$.

Suppose for instance that a disambiguation $\delta$ is antecedently in place in the setting for my utterance of (3), which is either silent when it comes to 'bat', or which associates it with sticks. That is, metaphorically, suppose that I am uttering (3) out of the blue or at Yankee Stadium. According to $(\mathrm{g})$, the verdict yielded by $\delta$ on 'bat', if any, is

\footnotetext{
10 Note that my account focuses on a case of particular interest, namely the sort of reflexive scenario in which, informally speaking, the disambiguation of $S$ is achieved by the utterance of $S$ itself. That is clearly not equivalent to the notion that this is the only source of disambiguation I admit. More interestingly, it also does not amount to the idea that no utterance of sentences other than $S$ may have a bearing on $S$ 's disambiguation, as in 'I bought a bat; my sister loves these animals' (thanks to an anonymous referee for suggesting this example). Especially due to my focus on disambiguating parentheticals in the latter parts of this essay, I leave these independently important generalizations aside.
} 
removed, and the result is enriched with the updated disambiguation engendered by (3), namely the zoological reading of that term. In a nutshell: other things being equal, if no previous clues were present that hinted at a disambiguation of 'bat', an utterance of (3) puts things in order. And even if contrasting hints had been in place, as in a conversation about baseball, (3) provides overt signals of a shift in disambiguating procedures.

The proposal in (d) can now be rectified: given a state $\sigma=\langle\langle\lambda, \delta\rangle, \beta\rangle$ (h) $\sigma\left[u_{S}\right]=<<\lambda, \psi^{S}>, \beta \cap \psi^{S}(\lambda)(S)>$

(second superscript on $\psi$ omitted). For instance, an utterance of (3) enriches $\beta$ with $\psi^{(3)}(\lambda)(3)$, that is, with the proposition that pipistrelles locate obstacles by echolocation.

A further English example may be appropriate, both as an additional informal clarification of (h) and as a hint towards further aspects of disambiguation. Take.

(4) bats are furry animals and banks are for money

and a state $\sigma$ such that $\beta$ includes common-sense beliefs about pipistrelles and all the rest, and such that $\delta$ yields, say, the religious meaning of the (here, irrelevant) word 'icon' and the stick meaning for 'bat', as in $\delta=\left\{\delta_{i c o n}, \delta_{b a t}, \ldots\right\}$. According to $(\mathrm{g})$, since the sentential form (4) contains 'bat', an utterance of (4) 'instructs' to subtract $\delta_{b a t}$ from $\delta$. What is added to the result is the utterance-disambiguation $v$ that maps 'bat' with its zoological sense and 'bank' with its financial meaning, thereby obtaining $\left\{\delta_{\text {icon }}, v_{\text {bat }}, v_{\text {bank }}, \ldots\right\}$. So, according to $(\mathrm{h}), \sigma\left[u_{(4)}\right]$ is a state containing (i) that disambiguation, and (ii) the intersection of $\beta$ with the proposition that pipistrelles are furry animals and that financial institutions are for money.

This has a noteworthy consequence: given normal common-sense background beliefs, the effects achieved by (4) on $\beta$ are trivial, since the desired interpretation has been obtained precisely on the basis of a process grounded on the idea that the aforementioned proposition was already part of common belief. This is as it should be: an utterance of (4) is indeed informationally idle against the shared background of minimally informed speakers. And yet, utterances of trivialities such as (4) are not inevitably out of place in the economy of conversation. In particular, their effects on an antecedent state $\sigma$ have to do with the replacement of $\delta$ with $\psi^{(4)}$. Informally speaking, that is, some utterances are put forth not with the primary aim of affecting common belief, but with the objective of constraining how certain utterances are to be interpreted. ${ }^{11}$

The applications of my formal apparatus to particular conversational instances will need to confront a variety of independent issues related to the idiosyncratic aspects of the examples under study. For concreteness' sake, I thus elect a particular phenomenon as my favourite implementation of the aforementioned mechanisms of disambiguation, the case of disambiguating parentheticals such as (1) and (2) at the beginning of this essay. Before I turn to this task, I provide a brief (and relatively negotiable) background on supplements in the next section.

\footnotetext{
11 This idea may have further repercussion on a variety of only superficially unrelated debates. An anonymous referee suggests an intriguing phenomenon, the idea of metalinguistic negotiation discussed (primarily in relation to legal discourse) in Plunkett and Sundell (2014) and (2021).
} 


\section{Supplements and disambiguating parentheticals: informal preliminaries}

Supplements have received a great deal of attention in linguistics. Classic examples include so-called non-restrictive relative clauses, as in.

(5) I spent part of every summer until I was ten with my grandmother, who lived in a working-class suburb of Boston (Potts, 2004: p. 6)

and nominal appositives such as.

(6) Ames, a successful spy, is now behind bars (Potts, 2004: p. 90),

or for that matter.

(7) bats, the topic of John's dissertation, have been in decline recently.

A few remarks on these examples suffice for my purposes here. The first has to do with the widespread sense that the informational contribution of a supplement is, in some sense, that of an aside. So, supplements are 'expressions that permit speakers to comment upon their assertions, to do a bit of editorializing in the midst of asking questions and imposing demands' (Potts, 2004: 8). For instance, in the case of (5), the proposition that the speaker's mother lived in a suburb of Boston is a commentary appended as an amplification of (5)'s main claim, namely that the speaker spent the summers of her youth with her grandmother. Similarly (taking on board the obvious interpretations of the nominal appositives respectively as in 'Ames was a successful spy' and 'bats are the topic of John's dissertation'), (6) and (7) say that Ames is behind bars and that bats have been in decline recently, together with the 'appended amplifications' that Ames was a successful spy and that bats are the topic of John's dissertation. ${ }^{12}$

The pre-theoretic idea of 'being an aside' is unquestionably worthy of theoretical reflection, and it has received a great deal of attention in the current literature on conversational structure. ${ }^{13}$ Yet, I briefly touched upon this apparent peculiarity of the appositives in (5)-(7) merely to note that, for my purposes, these independently important enrichments of our picture of conversation are of no immediate concern. And so, for pedagogical perspicuity, I continue with a simple-minded understanding of $\beta$ as an unstructured collection of propositions, occasionally reminding the reader of the independent adjustments needed whenever certain peculiarities of supplements come to the foreground. As a result, in my simple-minded approach, one way or

\footnotetext{
12 What I am simple-mindedly 'taking on board' blatantly bypasses the delicate issues pertaining to the syntactic and compositional regularities governing appositives (for a sense of the controversy surrounding these issues see for instance the contrast between McCawley, 1998 and Potts, 2004). Presenting and motivating my favourite view on these matters would take me well beyond the limits of this essay, especially in light of the fact that the aforementioned uncontroversial pre-theoretic intuitions about content (such as the notion that, among other things and in some special sense, (6) is also associated with the claim that Ames was a successful spy) suffice for my purposes in what follows.

13 Various conceptual tools have been mobilized for the theoretical analysis of this intuition, ranging from Potts' divide between at-issue and non-at-issue content, to more detailed distinctions between the different discursive roles that may be played by an utterance; see among many Roberts (1996), Asher and Lascarides (2003), Potts (2004), Amaral et al. (2007), Portner (2007), and Anderbois et al. (2015).
} 
another, utterances of (5), (6), or (7) simply propose to update $\beta$ as in $\beta \cap \pi_{1} \cap \pi_{2}$, where $\pi_{1}$ and $\pi_{2}$ are the aforementioned propositions. Preliminarily, then, given a sentential form $S$ of the form $M(A), M$ a 'main sentence' and $A$ an 'appositive',

(i) $\sigma\left[u_{S}\right]=<<\lambda, \delta>, \beta \cap \delta(\lambda)(M) \cap \delta(\lambda)(A)>$.

The reason for my overtly casual attitude here has to do with the fact that what matters for my topic are not the phenomena exemplified by (5), (6), or (7) per se, but rather the contrast between the effects of typical utterances of, say, (5), (6), or (7), and the outcomes engendered by the cases mentioned at the beginning of this essay, namely.

(1) bats, the furry animals, are not easy to find,

(2) I bought a bat (a pipistrelle),

or for that matter.

(8) bats (pipistrelles) are not easy to find, but you can get a bat (a hitting device) anywhere.

Assume the obvious disambiguation $\delta$ of 'bat' in the zoological sense. Then, according to a simple-minded application of (i) to, say, (1), what results from an utterance of that sentence is $\beta \cap \delta(\lambda)$ (that pipistrelles are not easy to find $) \cap \delta(\lambda)$ (that pipistrelles are furry animals), where $\beta$ is the conversants' antecedent common belief. Still in any common scenario, that pipistrelles are furry animals may safely be assumed to have already been part of $\beta$, the mutual cognitive arsenal of any minimally informed speaker. Propositional update, then, would effectively yield merely $\beta \cap \delta(\lambda)$ (that pipistrelles are not easy to find), a propositional outcome that fails to record any conversational difference between (1) and its simpler companion 'bats are not easy to find'.

And yet, clearly, (1), (2), and (8) do not seem to be conversationally on a par with their parenthetical-free counterparts. Indeed, figuratively speaking, the very banality of the content of those parentheticals is precisely what holds the key to the explanation of their role - that is, of the intuitive notion that, in this case, what must be at issue is a disambiguation of 'bat' in the zoological sense, rather than in the sense of 'stick'. In a nutshell: the point of the parentheticals is not the enrichment of common belief, but rather the indication of the conversationally appropriate disambiguation. I thus refer to (typical occurrences of the parentheticals in) (1), (2), and (8) as disambiguating parentheticals. I proceed to a clarification of my vague and informal preliminaries about these affairs in the next section.

\section{Disambiguating parentheticals and disambiguation update}

Suppose I say (1). As a part of tokening (1), I am also presenting to my conversants.

(9) bats are furry animals,

namely the material embedded as a parenthetical. In abstracto, this sentential form may be associated (according to $\lambda$, here for simplicity's sake English) with different claims, depending on the choice of $\delta_{b a t}$. And yet, the considerations about (3) from 
section three seem even more obviously relevant now: barring the preposterous claim that sticks are furry animals, what must be at issue is the zoological sense of 'bat', that is, a disambiguation $\delta_{b a t}$ such that $\delta_{b a t}(\lambda)(9)$ is the proposition that pipistrelles are furry animals.

That this disambiguating choice is, as I colloquially put it, obvious is apparently the positive side of the conundrum sketched towards the end of section four: although the proposition that pipistrelles are furry animals is surely nothing to write home about, the disambiguating effects achieved by (9) are conversationally noteworthy. Indeed, they are noteworthy precisely insofar as they naturally steer our interpretive decisions in the direction of the disambiguation appropriate for the main clause, that is, in the case of (1),

(10) bats are difficult to find.

Now, clearly, what is at issue is the not at all trivial proposition that pipistrelles, rather than wooder sticks, are rare.

The general ideas from section three, then, are naturally applicable to this case. Consider an antecedent state $\sigma=\langle\langle\lambda, \delta\rangle, \beta\rangle$ where no resources are in place for the disambiguation of 'bat', or, more dramatically, where $\delta_{b a t}$ yields the wooden stick sense of the term. In my metaphor, that is, imagine an out-of-the blue conversation or an exchange taking place at Yankee stadium. According to $(\mathrm{g})$, the updated disambiguation $\psi^{(9)}$ achieved by (9) disregards any previous disambiguation of 'bat', if any was at all in place, and 'instructs' to interpret that expression as in $v^{(9)}$ : the disambiguation $\delta$ such that $\delta(\lambda)(9)$ clashes with antecedent common belief is disregarded, leaving only the zoological sense intact. The result is a state whose common belief is $\beta \cap \psi^{(9}(\lambda)(9) \cap \psi^{(9}(\lambda)(10)$, that is, given the obvious traits of $\psi^{(9}(\lambda)(9)$, the common belief $\beta \cap \psi^{(9}(\lambda)(10)$, i.e., the enrichment of $\beta$ with the proposition that pipistrelles are difficult to find.

To generalize, conceive for simplicity's sake of affairs such as (1) as being of the form.

(j) $S=M_{1 \ldots n}\left(A_{i \ldots j}\right)$

where $M$ (the 'main clause') is a sentential form containing the ambiguous lexical forms $s_{1} \ldots s_{n}$ and $A$ (the 'nominal appositive') is a sentential form containing the ambiguous lexical forms $s_{i} \ldots s_{j}$. Then.

(k) $\sigma\left[u_{S}\right]=<<\lambda, \psi^{A}>, \beta \cap \psi^{A}(\lambda)(M) \cap \psi^{A}(\lambda)(A)>$.

This approach, as I am about to explain, reaches the apparently desired outcomes when it comes to cases such as (1), (2), or (8).

For one thing, the suggestion in $(\mathrm{k})$ reaches importantly different outcomes than those appropriate for straightforward supplements such as (5) and its ilk. Those supplements effect a standard update on $\beta$, since (in my simplified picture) $\sigma\left[u_{(5)}\right]=$ $<\left\langle\lambda, \delta>, \beta \cap \pi_{1} \cap \pi_{2}\right\rangle$, where $\pi_{1}$ and $\pi_{2}$ are the propositions mentioned in section four. On the other hand, the update on $\beta$ engendered by, say, (1) is indifferent to the parenthetical's contribution, that is, to the proposition that pipistrelles are furry animals, since that proposition is already part of $\beta$. This, in turn, entails an outcome consistent with the intuition that the propositional point of a disambiguating parenthetical is that of enriching common belief merely with the claim encoded in its main 
clause. Indeed, given the definition of $\psi^{A}$, this disambiguation is obtained by disregarding disambiguations responsible for non-trivial additions to $\beta$, so that $\beta \cap \psi$ ${ }^{A}(\lambda)(M) \cap \psi^{A}(\lambda)(A)=\beta \cap \psi^{A}(\lambda)(M)$.

A second related consequence is most profitably highlighted by returning to the fully superscripted format for the disambiguating update in question, namely ' $\psi{ }^{A, \sigma}$ ': the choice of the appropriate disambiguation depends on an aspect of the antecedent state $\sigma$, that is $\beta_{\sigma}$. Colloquially, the point is that obvious supplements are best suited for the role of disambiguating parentheticals. This seems correct. Intuitively, the divide between cases such as (1) and (7), repeated here.

(1) bats, the furry animals, are not easy to find

(7) bats, the topic of John's dissertation, have been in decline recently

hinges on the epistemic status of John's dissertation topic. And so, (1) is a better candidate than (7) for disambiguating purposes, but it is so only given the assumption that certain propositions, such as those pertaining to John's research interests, are more likely to be informative than the notion that bats are furry animals.

A third related issue also deserves to be emphasized. At least according to a natural understanding of the comments from the foregoing paragraphs, the divide between runof-the-mill supplements and disambiguating parentheticals is pragmatic, in the sense of emerging from an approach to conversational exchanges. So, notwithstanding my occasional description of certain sentences as involving disambiguating parentheticals, what are of interest are the updates which their utterances engender on particular conversational states. Utterances of (1) may well be more likely candidates than (7) for disambiguating purposes, but the difference is not semantic, or, for that matter, syntactic. In abstracto, then, the effects achieved by both sentences follow a parallel pattern. Yet, in the case of (7) and given an antecedent disambiguation $\delta$, a pair of presumably interesting propositions enter common belief, namely that $\delta(\lambda)$ ( 'bats') have been in decline and are the topic of John's dissertation, but no noteworthy effects on $\delta$ are detectable. In (1), on the other hand, the content of the antecedent belief $\beta$ already includes the propositional contribution of the supplement, whose interesting role is fully reflected by its disambiguating effects.

As is often the case with pragmatic phenomena, their application to particular concrete scenarios remains a delicate business. For one thing, I suppose, utterances may, on certain occasion, invite a partial retraction, readjustment, or accommodation of $\beta$. And so, could we not utter (1) with the aim of expressing the beliefs that sticks are furry animals that are hard to find, thereby inviting you to modify our antecedent common beliefs? Perhaps, even though such a move would clearly not be conversationally optimal in the absence of spectacularly overt coordinating preliminaries:

(11) I am about to use 'bat' for these sort of things [holding a stick]. Here it comes: bats, the furry animals, are not easy to find.

In my apparatus, the identification of a disambiguating update is also a rather direct affair, grounded on the relationships between $\beta$ and the proposition expressed by the parenthetical. Some conversational exchanges may follow a more roundabout pattern. For instance, I may say.

(12) bats, the exemplars of Chiroptera, are difficult to find. 
Disambiguating effects may well be in the air, even if it is not antecedently assumed that bats belong in the order Chiroptera, and indeed even if the form 'Chiroptera' is not part of our antecedent common competence. That sounds like one of those fancy names for zoological classification, you may reason. What must matter is the zoological reading for 'bat', you conclude. ${ }^{14}$ This sort of reasoning is grounded on independent additional phenomena, in particular having to do with the pragmatic repercussions of register. I simply leave them aside as independently interesting epicycles external to, but compatible with my general approach.

\section{Conclusion and the road ahead}

In this essay, I presented a general framework for disambiguation, and I applied it to the case of disambiguations achieved by virtue of uttering certain sentences as part of a conversational exchange, as in the case of (3) from section three. In section five, I focused on an interesting application of this framework, the case of disambiguating parentheticals, and I proposed an analysis of the contrast between run-of-the mill appositives, such as (7), and disambiguating parentheticals such as (1).

As repeatedly mentioned, my approach aimed at highlighting certain interesting traits of disambiguation in discourse, but explicitly steered clear from a fully-fledged analysis of that phenomenon in its entirety. And so, disambiguations can surely be achieved in ways other than those depicted in section three, and, even more obviously, without the help of disambiguating parentheticals. Indeed, as I gestured in the final paragraph of section five, even cases of this latter type may appeal to disambiguating procedures other than those explicitly depicted in $(\mathrm{k})$. The issues confronting a general theory of disambiguation, then, are uncontroversially delicate. My analysis, being focused on a particular, albeit especially intriguing, disambiguating procedure is thus insensitive to a variety of independently motivated complications-for instance, having to do with other, and possibly conflicting disambiguating resources, with the special status of appositive content, and with more besides.

Still, the outcomes are promising. In the spirit of an open-ended conclusion, I mention a few phenomena that seem to be naturally amenable to analyses ensuing from appropriate extensions of my framework (over and above those to which I have already alluded in some of the footnotes). For one thing, with opportune adjustments, the study of disambiguation seems to be naturally applicable to cases of homonymyresolution, with a parallel extension to parenthetical instances such as.

(16) Armstrong (the cyclist) is in trouble, but Armstrong (the astronaut) is a hero.

By the same token, extensions to structural ambiguity, ellipsis, or anaphora may not be hard to come by, as in the parenthetical clarification of the pronoun in.

(17) Carroll's objection is that in characterizing this practice Levinson is making empirical claims and he, Carroll, is not convinced ... (Lamarque, 2008: p. 128). ${ }^{15}$

\footnotetext{
14 Consider also the case of directly linguistically oriented parentheticals, as in 'bats, in the zoological sense of that term, are not easy to find'.

15 A difficult but interesting further phenomenon has to do with instances of so-called desemanticized occurrences, as in 'bachelors are either unmarried males or holders of a first degree', or, for a case closer
} 
The adaptations of my framework to these and other phenomena will need to be judged on their own merits. The enrichment of the representation of a conversational state with medium-oriented affairs such as $\lambda$ and $\delta$ is nevertheless a promising first step towards a fuller understanding of an utterance's conversational effects.

Open Access This article is licensed under a Creative Commons Attribution 4.0 International License, which permits use, sharing, adaptation, distribution and reproduction in any medium or format, as long as you give appropriate credit to the original author(s) and the source, provide a link to the Creative Commons licence, and indicate if changes were made. The images or other third party material in this article are included in the article's Creative Commons licence, unless indicated otherwise in a credit line to the material. If material is not included in the article's Creative Commons licence and your intended use is not permitted by statutory regulation or exceeds the permitted use, you will need to obtain permission directly from the copyright holder. To view a copy of this licence, visit http://creativecommons.org/licenses/ by $/ 4.0 \%$.

\section{References}

Amaral, P., Roberts, C., \& Allyn Smith, E. (2007). Review of the logic of conventional implicatures by Chris Potts. Linguistics and Philosophy, 30, 707-749.

Anderbois, S., Brasoneanu, A., \& Henderson, R. (2015). At-issue proposals and appositive impositions in discourse. Journal of Semantics, 32, 93-138.

Asher, N., \& Lascarides, A. (2003). Logics of conversation. Cambridge University Press.

Barwise, J (1988). Three views of common knowledge. In: TARK 88 proceedingsof the $2^{\text {nd }}$ conference on theoretical aspects of reasoning about knowledge. pp. 365-379.

Clark, H., \& Catherine, M. (1981). Definite reference and mutual knowledge. In A. K. Joshi, B. L. Webber, \& I. A. Sag (Eds.), Elements of siscourse understanding (pp. 10-63). Cambridge University Press.

Farkas, D., \& Bruce, K. (2009). On reacting to assertions and polar questions. Journal of Semantics, 27, $81-118$.

Kracht, M. (2007). The emergence of syntactic structure. Linguistics and Philosophy, 30, 47-95.

Lamarque, P. (2008). The philosophy of literature. Wiley.

McCawley, J. (1998). The syntactic phenomena of english. Chicago University Press.

Plunkett, D., \& Sundell, T. (2021). Metalinguistic negotiation and speaker error. Inquiry, 64, 142-167.

Plunkett, D., \& Tim, S. (2014). Antipositivist arguments from legal thought and talk: The metalinguistic response. In G. Hubb \& D. Lind (Eds.), Pragmatism, law, and language (pp. 56-75). Routledge.

Portner, P. (2007). Instructions for interpretation as separate performatives. In K. Schwabe \& S. Winkler (Eds.), On information structure meaning and form: Generalizations across languages (pp. 407-425). John Benjamins.

Potts, C. (2004). The logic of conventional implicature. Oxford University Press.

Potts, C. (2007). The dimensions of quotation. In C. Barker \& P. Jacobson (Eds.), Direct compositionality (pp. 405-431). Oxford University Press.

Predelli, S. (2013). Proper names: A millian perspective. Oxford University Press.

Roberts, C. (1996). Information structure in discourse: Towards an integrated formal theory of pragmatics. In: OSU Working papers in linguistics 49: Papers in semantics 49: pp. 91-136.

Seppänen, A. (1974). Proper names in english. A study in semantics and syntax. Publications of the Department of English Philology, University of Tampere.

Stalnaker, R. (1999). Context and content. Oxford University Press.

Stalnaker, R. (2014). Context. Oxford University Press.

Publisher's Note Springer Nature remains neutral with regard to jurisdictional claims in published maps and institutional affiliations.

Footnote 15 continued

to that mentioned in the previous footnote, 'Cambridge is an asylum in both senses of the word'. The first example is attributed to Seppänen 1974 in Predelli (2013): p. 161; the latter is generally credited to A. E. Housman. 